\title{
Green Synthesis of Silver Nanoparticles using Neem and Collagen of Fish Scales as a Reducing and Stabilizer Agents
}

\author{
Mustafa Mudhafar ${ }^{1,2, *}$, Ismail Zainol ${ }^{2}$, H.A. Alsailawi ${ }^{1}$ and C. N. Aiza Jaafar ${ }^{3}$ \\ ${ }^{1}$ Department of Anesthesia and Intensive Care Techniques, Faculty of AL, Tuff Collage, Karbala, 56001, Iraq \\ ${ }^{2}$ Department of Chemistry, Faculty of Science and Mathematics, Universiti Pendidikan Sultan Idris, Proton City, 35900 Tanjung Malim, \\ Perak, Malaysia, ${ }^{3}$ Department of Mechanical and Manufacturing Engineering, Faculty of Engineering, Universiti Putra Malaysia, 43000 \\ Serdang Selangor, Malaysia. ${ }^{4}$ Department of Biology, Faculty of Science and Mathematics, Universiti Pendidikan Sultan Idris, Proton City, \\ 35900 Tanjung Malim, Perak, Malaysia.
}

Received: March 16, 2020; Revised: Dec 2, 2020; Accepted: Dec 21, 2020

\begin{abstract}
This study was conducted to synthesize and characterize silver nanoparticles (AgNPs) using a rapid green synthesis approach. Antibacterial properties of AgNPs were evaluated. Extract of Melia Dubia (Neem) and collagen produced from the fish scales were used as reducing and stabilizer agents respectively. Uv-Vis Spectroscopy, Scanning Electron Microscopy (SEM), Energy-Dispersive X-Ray Spectroscopy (EDX), Fourier-Transform Infrared Spectroscopy (FTIR), and Dynamic Light Scattering (DLS) were used to characterize the synthesized AgNPs. Evaluation of the synthesized AgNP's antibacterial activity was conducted, with Staphylococcus Aureus (S. aureus) as Gram-positive and Escherichia Coli (E. coli) as the negative bacteria. The peak of absorbance for the synthesized AgNPs was at $454 \mathrm{~nm}$, indicating conformed AgNPs. SEM image showed semi-evenly distributed rod shapes. The EDX data revealed presence of the metallic silver. Meanwhile, FTIR analysis indicated presence of $\mathrm{C} 2 \mathrm{H} 2, \mathrm{C}=\mathrm{O}, \mathrm{N}-\mathrm{H}$ groups. DLS showed an average size of $437.6 \mathrm{~nm}$. XRD showed calculation for particle size using $\mathrm{d}=\mathrm{K} \lambda / \beta \cos \theta$. Average size of $\mathrm{AgNPs}$ was $141.81 \pm 5 \mathrm{~nm}$. AgNPs also displayed tangible antibacterial activity towards the S. aureus and pathogenic E. coli. AgNPS were successfully synthesized and evaluated for their antibacterial properties. The outcomes being the multifaceted biological activities alongside application of biocompatible green nanoparticles, is discoverable in the field of Nanomedicine.
\end{abstract}

Keywords: Silver nanoparticles, Fish Scales Collagen (FsCol), Melia Dubia (neem), Antibacterial

\section{Introduction}

AgNPs are common nanoparticles, vastly used in cleaning agents, commercial industry, food storage, healthcare products, packing and textile coating. Their antimicrobial property against bacteria, fungi, and viruses, makes them suitable for several environmental applications besides being used extensively in products specific to biomedical purposes such as topical creams, antiseptic sprays, and wound dressings. This is due to their capability in disabling a microorganism's enzymatic activities by disrupting the membrane of the pathogenic organisms (Haghighi et al., 2017; Pugazhendhi et al., 2018).

Many techniques were introduced to synthesise AgNPs, such as thermal decomposition, chemical reduction of silver ions $(\mathrm{Ag}+)$, pyrolysis microwave irradiation, electrochemical methods, and laser ablations (Beyene et al., 2017; Sundarrajan et al.,2019). Among all, the most traditional way to synthesize AgNPs is via chemical reduction technique. The agents commonly used lessen the chemical are sodium borohydride and sodium citrate (Ojo et al., 2017). Traditional chemical synthesis stipulates the necessity to have metal precursor, reducing agents and capping/stabilizing agents to prevent vigorous spreading of the nanoparticles (Chowdhury et al., 2015). The traditional ways are claimed to be more effective in controlling distribution and reproducibility of the nanoparticle size as they require greater input of energy alongside presence of toxic chemicals. Under certain circumstances, it may require occurrence of controlled pressure and temperature. This may lead to higher cost and environmental contamination.

"Green chemistry" was introduced as an economically feasible option and is known to be a biogenic way to synthesize metallic nanoparticles and AgNPs (Ahmed et al., 2019). This approach is known to be a biogenic synthesis of the metallic nanoparticles. This approach is reputable for its simplicity, environmental-friendliness, cost-effectiveness and scale-up efficiency. The procedure is carried out at room temperature and pressure, within aqueous ambiance, without organic solvents (Sportelli et al., 2018). It is also conducted via biological systems, which includes algae, bacteria, plants, fungi, diatoms, and yeast. The simplicity of the biogenic way made it possible for plant extracts to be used to eradicate the procedure of sustaining cell cultures. Metallic nanoparticles that are based on plant-mediated synthesis are also less biohazardous (Mudhafar et al., 2020).

Recently, plant extracts have gained much interest. They are commonly used as agents to reduce and cap

\footnotetext{
* Corresponding author e-mail: Almosawy2014@gmail.com.
} 
metallic nanoparticles mainly due to their advantageous potentials which include fast, simple, economically feasible green technique, and environmentally-friendly nature (Ajitha et al., 2016). The phytochemicals of the plants play the role of tough reducing agent, resulting in development of capped nanoparticles. This signifies that plant extract is a one-step natural capping and reducing agent, reducing not just the cost but the use of chemical reagents too (Govindarajan et al., 2017). In addition, chemical compounds are replaced with phytochemicals' surroundings, while organic solvents are replaced by aqueous surroundings (Jyoti et al., 2016). Today, distinct parts of plants, like its fruits, bran, bark, flowers, and leaf, are used in metallic nanoparticle synthesis (Shaik et al., 2018) as they possess medicinal value attributed to presence of amino acids, phenolic compounds, such as polyphenols, flavonoids, terpenoids, and vitamins (Mudhafar et al., 2019).

Neem is a part of the Melia genes belonging to the Meliaceae family. It is greatly distributed in India and Malaysia. The Indian neem have been extracted and used to produce nano-silver, showing good result when AgNO3 was used for anticancer activity. Kathiravan et al., (2014), have synthesized the AgNPs for anticancer. However, there has yet to be any report of AgNPs synthesis from neem leaves and its antimicrobial activity from neem extract.

Collagen is a known biocompatible polymer that is water-soluble, and synthetic, evidenced by its broad use for biomedical purposes. Among such purpose includes the use of nanoparticles' coating to enhance spreading of nanoparticles in an aqueous medium and their penetration into the mucus layer. Furthermore, collagen can guard nanoparticle against immune system clearance by avoiding the opsonization of nanoparticle in the blood, lessening the toxicity of nanoparticles, and increasing the lifetime of the nanomaterial within the circulation system (Sawadkar et al., 2019; Mudhafar et al., 2020). Additionally, collagen can also be used as a stabilizing agent (Tarannum et al., 2015). For the purpose of this study, Fish Collagen was used as safe stabilizing agent for AgNPs.

The AgNPs' biogenic synthesis was studied with the one-pot green protocol because of its advantageous traits. Meanwhile, the reducing agent used was from neem extracts. To enhance the capability of the green neem that synthesized the AgNPs, the nanoparticles were stabilized with collagen. Different analytical methods were conducted to characterize the AgNPs. The classification of the AgNPs' infusion of antimicrobial activity towards a few Gram-positive and Gram-negative pathogenic bacterial strain was conducted based on vitro evaluation. Outcome from a simple green protocol revealed that the commercial green neem extract led to successful development of plant-mediated AgNPs. Furthermore, the nanoparticles' antibacterial activity against varying strains of bacteria was shown at concentration. These support outcomes from the previous study, where biosynthesis of nanometals had strong antibacterial effects.

\section{Materials and methods}

\subsection{Materials}

$\mathrm{AgNO}_{3}$ was obtained from Bendosen Company. Neem leaves were sourced locally from Perak, Malaysia. Collagen was extracted from fish scales and were produced in the Chemistry Department laboratory, UPSI, Perak, Malaysia. The broth and nutrient agar were bought from Merck.

\subsection{Methods}

\subsubsection{Neem leaf preparation}

Fresh neem leaves were collected from Perak. The leaves were cleaned several times to eradicate dust and fungi after which it was sun-dried for one week to completely remove moisture. The dried neem leaves were then crushed and converted to powder form. A total of $10 \mathrm{~g}$ powder was extracted with $100 \mathrm{ml}$ distilled water in a 250 $\mathrm{ml}$ conical flask. Subsequently, the powder was heated for 10 minutes, cooled and filtered to obtain a crude extract before being incubated at $4{ }^{\circ} \mathrm{C}$.

\subsubsection{Green synthesis of AgNPs}

$1 \mathrm{mM}$ of $\mathrm{AgNO}_{3}$ was dissolved in $90 \mathrm{ml}$ distilled water and the solution formed was transferred into a $250 \mathrm{ml}$ conical flask. $10 \mathrm{ml}$ of crude extract was then added into the conical flask and stirred thoroughly. Then, $0.1 \mathrm{~g}$ of collagen was added into the conical flask and the mixture was again stirred thoroughly. UV-Vs Spectroscopy was then utilized to analyses development of AgNPs. The solution containing AgNPs was centrifuged for 10 minutes at a rate of $14000 \mathrm{rpm}$. Meanwhile, the supernatants were discarded, and the NP portion was sterilized in distilled water. This aforementioned step was repeated three times to eradicate unused biomaterial and impurity. The freezedrying method was used to obtain dry particles for the purified sample of dry particles (Joseph et al., 2015).

\subsubsection{Characterization of AgNPs}

Absorbance between 200nm and $800 \mathrm{~nm}$ was conducted using the UV-Vis Spectroscopy Agilent Cary 60 UV Spectrophotometer. EDX, SEM, and DLS were performed respectively via Bruker X Flash 6110, Nova Nanosem 450.

\subsubsection{Antibacterial activity}

Paper disk diffusion test was employed to investigate antibacterial activities of $E$. coli and $S$. aureus. Nutrient agar media was used to cultivate bacteria. $10 \mu \mathrm{g}$ of AgNPs was saturated in $1 \mathrm{ml}$ of distilled water and tested against the bacteria. Neem were also examined. Next, a total of 10 , 20, 40, and $80 \mu \mathrm{M} / \mathrm{ml}$ of AgNPs were dissolved in distilled water and poured onto a 6 mm disk filter [24]. Upon 24 hours incubation, inhibited zones were measured and the magnitude of antibacterial impacts on $S$. aureus and the $E$. coli were determined.

\section{Results and Discussion}

\subsection{UV-Vis Spectroscopy}

UV-Vis Spectroscopy was used to corroborate stability and development of AgNPs in a solution. Figure 1 displays the extinction spectra of AgNPs. As observed, the 
absorption band of synthesized AgNPs was $454 \mathrm{~nm}$. Many studies have documented absorbance of AgNPs, whereby peak of surface plasmon for AgNPs sized $<100$ $\mathrm{nm}$ was located between $400-450 \mathrm{~nm}$ (Rathod et al., 2016).

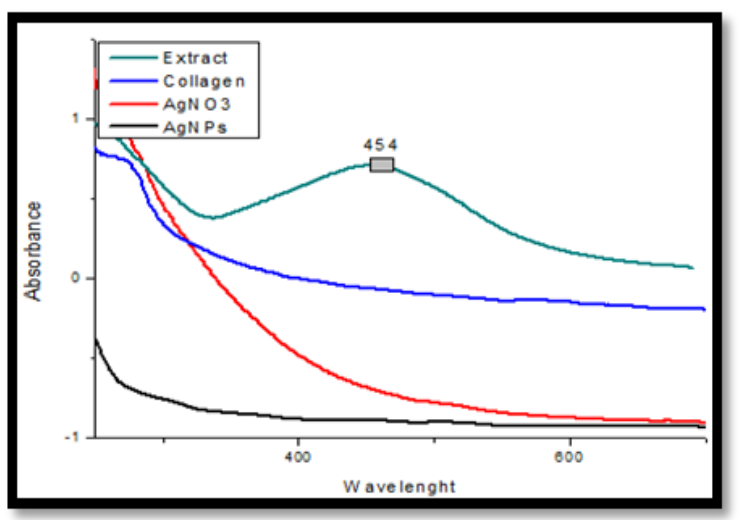

Figure 1. UV-Vis Spectroscopy of AgNPs, $\mathrm{AgNO}_{3}$ and $\mathrm{FsCol}$

\subsection{SEM}

SEM was utilised to classify the parts of synthesized AgNPs. SEM's image displays the AgNPs' constant spreading. It was discovered that the AgNPs had a rod-like shape with smooth morphology, almost the same shape as the AgNPs when using a microwave. Figure 2 displays SEM image such as that of the prepared powdered AgNPs. The magnification specification for SEM image was 100000x for $500 \mathrm{~nm}$ image size, which is a considerably big for AgNPs. Especially since the formation of AgNPs were considerably shorter compared to that mentioned in literature; that the size of the Silver Nanoparticles will decrease with increased formation time (Dehnavi et al., 2013).

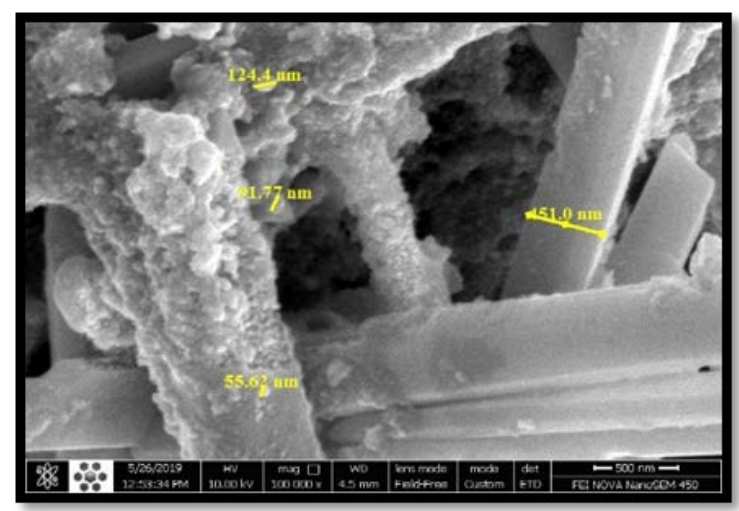

Figure 2. SEM for the morphology and measured of AgNPs

\subsection{FTIR}

FTIR was used to evaluate factional groups of fish collagen and neem leaves in comparison with factional groups of synthesized AgNPs. This is to gain insight on factional groups surrounding the silver nanoparticles. As shown in Figure 4, the FsCol reveals two major peaks, which are $3441 \mathrm{~cm}-1$ for the $\mathrm{NH}$ (amide A) and $1646 \mathrm{~cm}-1$ for CO stretching (amide I) (Hamidi et al., 2019). Peaks of the four miners were monitored at $1548 \mathrm{~cm}-1,1466 \mathrm{~cm}-1$, $1392 \mathrm{~cm}-1$, and $1242 \mathrm{~cm}-1.1548 \mathrm{~cm}-1-1466 \mathrm{~cm}-1$ refers to the $\mathrm{N}-\mathrm{H}$ bend coupled with $\mathrm{C}-\mathrm{N}$ stretch (Amide II), and $1392-1242 \mathrm{~cm}-1$ refers to the $\mathrm{N}-\mathrm{H}$ bending (Amide III) [23]. Neem leaves were characterized with two major peaks located at $3296 \mathrm{~cm}-1$ and $1629 \mathrm{~cm}-1$. The peak at $3296 \mathrm{~cm}-1$ refers to the stretch of $\mathrm{N}-\mathrm{H}$ and $\mathrm{O}-\mathrm{H}$ due to the presence of water, alcohols, and phenols (polyphenols) in neem extract (Philip, 2010). Amide I was observed at 1629 $\mathrm{cm}-1$, while miner peaks were observed at $2115 \mathrm{~cm}-1,618$ $\mathrm{cm}-1,480 \mathrm{~cm}-1$, and $333 \mathrm{~cm}-1$. At $2115 \mathrm{~cm}-1$, the bands appeared parallel to the present Alkyne group (C2H2) because of neem extract phytoconstituents (Mudhafar et al., 2019). At $618 \mathrm{~cm}-1$, the band matched the $\mathrm{C}-\mathrm{H}$ bonds in the phenolic rings while the band at $480 \mathrm{~cm}-1$ were attributed to the existence of alkyl halides.

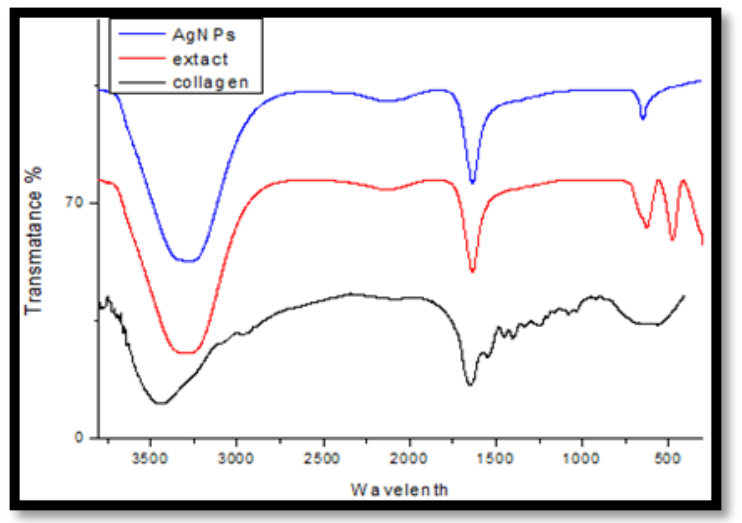

Figure 3. FTIR spectrum of FsCol, M. Dubia, and AgNPs

Among AgNPs, two major peaks were observed in the FTIR graph; $3315 \mathrm{~cm}-1$ and $1641 \mathrm{~cm}-1$. At $3315 \mathrm{~cm}-1$, the band is referring to the stretch of $\mathrm{O}-\mathrm{H}$ and $\mathrm{N}-\mathrm{H}$ (amide A), while at $1641 \mathrm{~cm}-1$, the band refers to the similar stretching of CO that belonging to amide I. Both these bands were observed in the fish collagen and neem extract. The band located at $2128 \mathrm{~cm}-1$, refers to alkyne group $\left(\mathrm{C}_{2} \mathrm{H}_{2}\right)$ from the neem extract phytoconstituents. At 1361 $\mathrm{cm}-1$, the band corresponds to $\mathrm{N}-\mathrm{H}$ bending (Amide III) from fish collagen. Two peaks at $608 \mathrm{~cm}-1$ and $689 \mathrm{~cm}-1$ indicates $\mathrm{C}-\mathrm{H}$ bonds in phenolic rings. Table 1 shows all details for AgNPs, plant extract and FsCol (Singh et al., 2016: Mudhafar et al., 2021).

Table 1: Factional groups in AgNPs in FTIR

\begin{tabular}{llll}
\hline Factional groups & \multicolumn{2}{l}{ Wavenumber cm } \\
\hline & FsCOL & Plant extract & AgNPs \\
\hline N-H (stretch) & $3446 \mathrm{~cm}^{-1}$ & $3296 \mathrm{~cm}^{-1}$ & $3301 \mathrm{~cm}^{-1}$ \\
Alkyne group $\left(\mathrm{C}_{2} \mathrm{H}_{2}\right)$ & -- & $2115 \mathrm{~cm}^{-1}$ & $2140 \mathrm{~cm}^{-1}$ \\
C=O Stretching (amide I) & $1646 \mathrm{~cm}^{-1}$ & $1629 \mathrm{~cm}^{-1}$ & $1668 \mathrm{~cm}^{-1}$ \\
N-H bends couple C-N & $1531 \mathrm{~cm}^{-1}$ & -- \\
stretch (Amide II) CN & & & \\
Amide III N-H bend & $1348 \mathrm{~cm}^{-1}$ & & $1366 \mathrm{~cm}^{-1}$ \\
Amide III N-H bend & $1242 \mathrm{~cm}^{-1}$ & & $1227 \mathrm{~cm}^{-1}$ \\
O-H & -- & $618 \mathrm{~cm}^{-1}$ & $634 \mathrm{~cm}^{-1}$ \\
Alkyl halides & -- & $480 \mathrm{~cm}^{-1}$ & $488 \mathrm{~cm}^{-1}$ \\
\hline
\end{tabular}

3.4. DLS

Zeta sizer shows the peak of AgNPs. Based on the pattern, the AgNPs synthesized through this technique had shown a 0.249 polydispersity index (PDI) with a Zeta average diameter of $437.6 \mathrm{~nm}$. The size of the zeta sizer measures is slightly bigger than the size of the particle as measured in SEM micrographs due to DLS method which measures the hydrodynamic radius (Ahmed et al., 2017). In addition, DLS also measures attached surface proteins, 
carbohydrates, and other cellular materials. However, they could not be kept in a vacuum under an electron beam as it can also be associated with the consistency of biosynthesis (Liu et al., 2019). The DLS graph of synthesized AgNPs showed two peaks, a strong peak at $480.7 \mathrm{~nm}$ with $98 \%$ intensity area and a weak peak at $5256 \mathrm{~nm}$ with $2 \%$ intensity area. Meanwhile, the Zeta average diameter was $437.6 \mathrm{~nm}$, as shown in Figure 5.

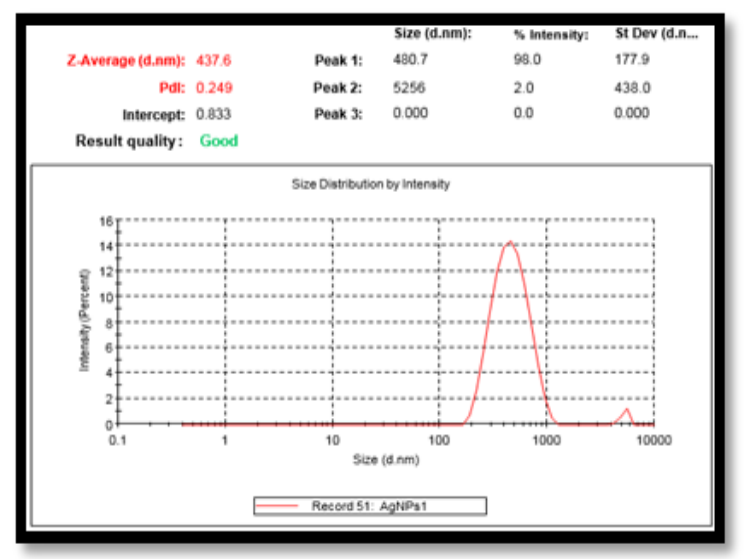

Figure 4. DLS analyses of AgNPs

\subsection{Antibacterial activities}

The AgNPs' antibacterial properties were recorded according to disk diffusion method. A ruler was used to measure the AgNPs' ability to prevent bacterial growth against both bacteria (Anandalakshmi et al., 2016). Distilled water was used for negative control, while ampicillin was used and tested against bacteria for positive control. Figure 5 shows the inhibition zones of $E$. coli and $S$. aureus bacteria. The distilled water inhibition appeared zero in both bacteria. The inhibition zones of the extract were 12.2 and $10.4 \mathrm{~mm}$, in contrast to E. coli and $S$. aureus, respectively. The inhibition zones of ampicillin were 28.6 for E. coli and 22.1 for S. aureus. Many AgNPs' concentrations resulted in good inhibition zones against both bacteria. The inhibition zone increased with AgNPs concentration. Table 2 shows details of AgNPs inhibition and control.

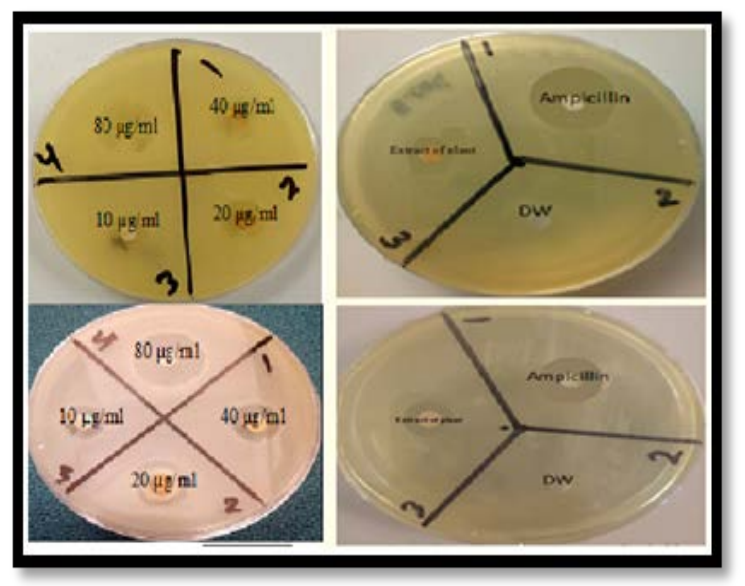

Figure 5. Antibacterial activities of A) AgNPs and control samples against E. Coli and B) AgNPs against S. Aureus

\section{Conclusions}

The characteristics of biosynthesis methods, such as safety, low cost, and environmental friendliness are deemed attractive by researchers. Green synthesis method was employed in this study to synthesize AgNPs from biological sources. Neem leaves were utilized as natural source. The use of UV-vis, FTIR, SEM, EDX, and DLS resulted in successful synthesis and categorization of AgNPs. The morphology and particle size were also investigated. These techniques revealed a rod shape with average sizes ranging from $150 \mathrm{~nm}$ to $430 \mathrm{~nm}$. The antimicrobial characteristics of the synthesized AgNPs were also examined using the Gram-positive and Gramnegative bacteria. To which it was discovered that AgNPs was highly efficient as an agent for microbes and has great potential for use as antibacterial agent within the medical field.

\section{Acknowledgement}

This study was partially supported by grant no. FRGS 2019-0147-103-02

\section{References}

Ahmed, S., Ahmad, M., Swami, B. L., \& Ikram, S. 2016. A review on plants extract mediated synthesis of silver nanoparticles for antimicrobial applications: a green expertise. $J$ adv Res, 7(1): $17-28$.

Ahmed, S., Saifullah, Ahmad, M., Swami, B. L., \& Ikram, S. 2016. Green synthesis of silver nanoparticles using Azadirachta indica aqueous leaf extract. J Rad Res appl Sci, 9(1): 1-7.

Ajitha, B., Reddy, Y. A. K., Reddy, P. S., Jeon, H. J., \& Ahn, C. W. 2016. Role of capping agents in controlling silver nanoparticles size, antibacterial activity and potential application as optical hydrogen peroxide sensor. RSC advances, 6(42): 3617136179.

Anandalakshmi, K., Venugobal, J., \& Ramasamy, V. 2016. Characterization of silver nanoparticles by green synthesis method using Pedalium murex leaf extract and their antibacterial activity. Appl Nanosci, 6(3): 399-408.

Beyene, H. D., Werkneh, A. A., Bezabh, H. K., \& Ambaye, T. G. 2017. Synthesis paradigm and applications of silver nanoparticles (AgNPs), a review. Sust Mat Technol, 13: 18-23.

Chowdhury, S., Yusof, F., Sulaiman, N., \& Faruck, M. O. 2017. The Aggregation Study and Characterization of Silver Nanoparticles. Solid State Phen, 263:165-169.

Dehnavi, A. S., Raisi, A., \& Aroujalian, A. 2013. Control size and stability of colloidal silver nanoparticles with antibacterial activity prepared by a green synthesis method. Synthesis and Reactivity in Inorganic, Metal-Organic, and Nano-Metal Chemistry, 43(5): 543-551.

Govindarajan, M., Kadaikunnan, S., Alharbi, N. S., \& Benelli, G. 2017. Single-step biological fabrication of colloidal silver nanoparticles using Hugonia mystax: larvicidal potential against Zika virus, dengue, and malaria vector mosquitoes. Arti Cells, Nanomedicine, and biotechnol, 45(7): 1317-1325.

Haghighi Pak, Z., Karimi, N., \& Abbaspour, H. 2017. Effects of Silver Nanoparticle Exposure on Growth, Physiological and biochemical Parameters of Dracocephalum moldavica L. Plant Phys, 7(4): 2173-2183.

Hamidi, A., Yazdi, M. E. T., Amiri, M. S., Hosseini, H. A., \& Darroudi, M. 2019. Biological synthesis of silver nanoparticles in Tribulus terrestris L. extract and evaluation of their photocatalyst, antibacterial, and cytotoxicity effects. Res Chem Intermid, 45(5): 2915-2925. 
Joseph, S., \& Mathew, B. 2015. Facile synthesis of silver nanoparticles and their application in dye degradation. Mat Sci Eng, B, 195: 90-97.

Jyoti, K., Baunthiyal, M., \& Singh, A. 2016. Characterization of silver nanoparticles synthesized using Urtica dioica Linn. leaves and their synergistic effects with antibiotics. J Rad Res Appl Sci, 9(3): 217-227.

Kathiravan, V., Ravi, S., \& Ashokkumar, S. 2014. Synthesis of silver nanoparticles from Melia dubia leaf extract and their in vitro anticancer activity. Spectrochimica Acta Part A: Mol Biomol Spectroscopy, 130:116-121.

Mudhafar M, Zainol I, Jaafar CNA, Alsailawi HA, Desa S. 2021. A review study on synthesis methods of Ag nanoparticles, considering a antibacterial property and cytotoxicity. Int J Drug Delivery Technol, 2:635-48.

Liu, Y., Kim, S., Kim, Y. J., Perumalsamy, H., Lee, S., Hwang, E., \& Yi, T. H. 2019. Green synthesis of gold nanoparticles using Euphrasia officinalisleaf extract to inhibit lipopolysaccharideinduced inflammation through NF- $\mathrm{KB}$ and JAK/STAT pathways in RAW 264.7 macrophages. Int J Nanomed, 14: 2945.

Mudhafar, M., \& Alsailawi, H. A. 2019. An Expression Study Profile of Proinflammatory Cytokines in Asthma Patient. J Asian Sci Res, 9(12): 227-234.

Mudhafar, M., Zainol, I., Desa, S 2019. and Che Nor Aziza Jaafar. "review of Phytochemistry for Polyalithia 14:119-147

Mudhafar, M., and Zainol, I. "Medical values, antimicrobial, and anti fungal activities of Polyalthia genus 2019." Int J Pharml Res, 11: $91-96$.

Mudhafar, M., Zainol, I., Jaafar, C. N., Alsailawi, H. A., Majhool, A. A., \& Alsaady, M. 2020. Phytochemical Screening and Characterization of Meliadubia Leaves Extract for Antimicrobial Activity against Escherichia coli and Staphylococcus aureus. Indian J Ecol, 47(2): 493-496.

Mudhafar M, Zainol I, Alsailawi HA, Aiza Jaafar CN. 2021. Synthesis and characterization of fish scales of hydroxyapatite/collagen-silver nanoparticles composites for the applications of bone filler. J Korean Ceramic Soc. 12:1-1.

Ojo, O.A., Oyinloye, B.E., Ojo, A.B., Afolabi, O.B., Peters, O.A., Olaiya, O., Fadaka, A., Jonathan, J. and Osunlana, O., 2017. Green synthesis of silver nanoparticles (AgNPs) using Talinum triangulare (Jacq.) Willd. leaf extract and monitoring their antimicrobial activity. J Bionanosci, 11(4): 292-296.
Pugazhendhi, A., Prabakar, D., Jacob, J.M., Karuppusamy, I. and Saratale, R.G., 2018. Synthesis and characterization of silver nanoparticles using Gelidium amansii and its antimicrobial property against various pathogenic bacteria. Microbial patho, 114: 41-45.

Rathod, D., Golinska, P., Wypij, M., Dahm, H. and Rai, M., 2016. A new report of Nocardiopsis valliformis strain OT1 from alkaline Lonar crater of India and its use in synthesis of silver nanoparticles with special reference to evaluation of antibacterial activity and cytotoxicity. Med Microbiol Immunol, 205(5): 435447.

Sawadkar, P., Sibbons, P., Ahmed, T., Bozec, L., \& Mudera, V. 2019. Engineering of a functional tendon using collagen as a natural polymer. ACS Biomat Sci Eng, 5(10): 5218-5228.

Shaik, M.R., Khan, M., Kuniyil, M., Al-Warthan, A., Alkhathlan, H.Z., Siddiqui, M.R.H., Shaik, J.P., Ahamed, A., Mahmood, A., Khan, M. and Adil, S.F., 2018. Plant-extract-assisted green synthesis of silver nanoparticles using Origanum vulgare L. extract and their microbicidal activities. Sustainability, 10(4): 913.

Singh, Priyanka, Yeon J. Kim, Chao Wang, Ramya Mathiyalagan, and Deok C. Yang. "Weissella oryzae DC6-facilitated green synthesis of silver nanoparticles and their antimicrobial potential." Art Cells, Nanomed Biotechnol, 2016; 44: 1569-1575.

Sportelli, Maria C., Maurizio Clemente, Margherita Izzi, Annalisa Volpe, Antonio Ancona, Rosaria A. Picca, Gerardo Palazzo, and Nicola Cioffi. "Exceptionally stable silver nanoparticles synthesized by laser ablation in alcoholic organic solvent $\mathrm{A}$ Physicochemical and engineering aspects 2018; 559: 148-158.

Sripriya, Ramasamy, and Ramadhar Kumar. "A Novel Enzymatic Method for Preparation and Characterization of Collagen Film from Swim Bladder of Fish Rohu (Labeo rohita). Food Nut Sci, 2015; 6: 1468

Sundarrajan, Priya, and Sangeetha Shetty. "Synthesis And Characterization Of Silver Nanoparticles Using Various Plant Extracts And Their Various Environmental Applications 2019; 5: 91.

Tarannum, Aafiya, Charuvaka Muvva, Ami Mehta, J. Raghava Rao, and N. Nishad Fathima. "Phosphonium based ionic liquidsstabilizing or destabilizing agents for collagen. RSC Advances, 2016; 6: 4022-4033. 